\title{
La politique du silence dans 1 Henry VI
}

\section{Catherine Lisak}

\section{(Q) OpenEdition \\ Journals}

Édition électronique

URL : http://journals.openedition.org/shakespeare/411

DOI : $10.4000 /$ shakespeare.411

ISSN : 2271-6424

Éditeur

Société Française Shakespeare

Édition imprimée

Date de publication : 1 novembre 1999

Pagination : 173-186

ISBN : 2-84269-331-0

Référence électronique

Catherine Lisak, « La politique du silence dans 1 Henry VI 》, Actes des congrès de la Société française Shakespeare [En ligne], 17| 1999, mis en ligne le 01 novembre 2007, consulté le 02 mai 2019. URL : http://journals.openedition.org/shakespeare/411 ; DOI : 10.4000/shakespeare.411 


\section{S H A K E S P E A R E \\ \& $\quad$ L $A \quad$ V O I X}

Société Française Shakespeare

Actes du Congrès de 1999

米米

Textes réunis et présentés par

Patricia DORVAL

publiés sous la direction de

Jean-Marie MAGUIN 


\section{LA POLITIQUE DU SILENCE DANS 1 HENRY VI}

Of Secrecie. Defi. : Secrecie is a faithfull humor, which strengthened by uertue, concealeth in despight of misfortune, those things which one knoweth may eyther propt his enemy, or preiudice his friend or country.

Silence is unsuspected, but much babling is treasonfull.

Secrecie breedeth seuritie. ${ }^{1}$

Très vite, la question principale dans 1 Henry VI est de savoir quel sens donner au silence : «What means this silence ?» demande Richard Plantagenet ${ }^{2}$. Cette interjection - qui reflète un embarras prêt à tourner à la détresse - constitue les premiers mots articulés par ce personnage qui entretient avec le silence et la voix un rapport à plus d'un égard difficile. En effet, on ne se met à l'entendre et à entendre parler de lui que vers la fin de l'acte II. Qui plus est, Shakespeare lui réserve cette apparition tardive dans une scène totalement an-historique, en contraste avec les scènes de bataille qui précèdent ou suivent. Shakespeare n'a pas jugé bon jusque-là de lui donner voix au chapitre. C'est dans la scène entièrement fictive $\mathrm{du}$ jardin du Templier (on note que le nom de la dynastie Plantagenet est souvent associé à des métaphores végétales) que Richard nous est enfin présenté comme celui qui cherche, d'entrée 
de jeu, à briser un silence qui lui insupporte et le lèse. Voici un personnage qui souffre non seulement de la voix tue des autres mais aussi de l'extinction de sa propre voix.

Le dramaturge n'est d'ailleurs pas seul à maintenir ce personnage si longtemps dans l'aphonie des coulisses. L'Histoire semble, elle aussi, l'avoir évincé de l'arbre généalogique des nobles et des exploits qui la forgent. Cela tient au fait que le jeune Plantagenet est fils de traître. Son père Richard, comte de Cambridge et frère d'Aumerle, fut autrefois impliqué dans un complot pour assassiner Henry $\mathrm{V}^{3}$ et pour cela non seulement frappé de mort civile mais déshérité de ses biens, de ses propriétés et de son titre. Rappelons que l'exhérédation touchait le traitre ainsi que toute sa famille et ses descendants, estimés tous à jamais de sang corrompu et en butte à un destin tourné en dérision. L'infamie du coupable représentait une contamination irrévocable, par laquelle père restait vivant dans le fils, atteint et souillé (attainted) par la trahison paternelle qui n'était pas simplement un crime en actes, mais un défaut spirituel et même génétique. La tare était de nature héréditaire, comme le souligne son rival le duc de Somerset, qui rappelle à tous l'inavouable numerus clausus qui exclut Richard Plantagenet de toute revendication. Ce ne sont pas ses rapports à la loi mais ses liens de chair et de sang qui maintiennent le jeune homme dans la déchéance ignoble et dans l'opprobre :

Som. Was not thy father, Richard Earl of Cambridge,

For treason headed in our late king's days?

And by his treason stand'st not thou attainted,

Corrupted, and exempt from ancient gentry?

His trespass yet lives guilty in thy blood;

And till thou be restor'd, thou art a yeoman.

$$
\text { (1 Henry VI, 1.4.90-5) }{ }^{4}
$$

En réalité, même si on savait la personne innocente, on ne pouvait rien pour elle aussi longtemps que l'attainder n'avait pas été levée. Et pourtant, Plantagenet ne se soumettra pas à cette loi mais exigera obstinément réparation. La première partie d'Henry $V I$ va représenter pour Plantagenet, bientôt le duc d'York, comme pour son fils après lui, le duc de Gloucester, la marche initiale pour regagner le titre perdu de noble puis de prétendant à la Couronne. 
Plantagenet ouvre la scène 4 de l'acte II en lachant un vocatif mal maîtrisé, qui mêle l'indignation au respect. Dans cet élan vocal, on l'entend qui vocifère sa frustration face au silence que lui oppose son entourage noble autour d'une question floue, dite «question de vérité». Ainsi les met-il au défi, pour les provoquer à confirmer une vérité restée non-dite et à rompre un silence qu'il reçoit comme de la malignité et une injustice personnelle :

Plan. Great lords and gentlemen, what means this silence? Dare no man answer in case of truth ?

Suff. Within the Temple Hall we were too loud ; The garden here is more convenient.

$$
\text { (1 Henry VI, II.4.1-4) }
$$

Plantagenet est un personnage que l'absence de voix, de part et d'autre, étouffe ; car le silence injurieux et scandaleux qui charge l'espace scénique se compose de paroles retenues et de voix tues. Suffolk justifie le parti pris du silence comme un choix d'ordre stratégique, comme une mesure de précaution et de protection, motivée par la crainte et la méfiance : la voix est réfrénée, de peur que, dans l'écho ou dans la rumeur, elle n'échappe de leur contrôle. Si l'agacement de Plantagenet souligne leur lâcheté - pour lui, se prononcer, c'est oser, encourir un risque (to dare) - Suffolk conçoit le silence comme une preuve de pouvoir, une forme de résistance, une expression politique de prudence.

Dans un édifice public, la voix ne porte pas de la même manière selon qu'on se trouve dans l'enceinte d'une vaste salle ou celle d'un jardin. Ainsi s'opposent l'intérieur sonore et l'extérieur intime; en contraste avec l'espace clos, où la voix, pourtant trop audible, devient exposée à une écoute suspecte qui recueille à mauvais escient le bruit qui court - et alors le silence seul abrite du danger - l'espace à ciel ouvert où la voix demeure contenue, et où l'abri du feuillage maintient toute parole à couvert, laissant ainsi la voie libre aux conversations les plus intimes, les plus secrètes, voire les plus périlleuses. Opposition, en somme, entre l'interdit et l'interdit: «Must your bold verdict enter talk with lords?» s'exclame Plantagenet. Dans 1 Henry VI on hésite souvent à engager un dialogue en public autour de la question de l'héritage de Plantagenet, celle-ci demeurant pour certains une question tabou, pour d'autres une vérité allant de soi.

Le jardin est un lieu de refuge où la voix n'est pas répercutée, où le secret n'exige plus le silence. Là, les personnages peuvent 
donc s'entretenir en confidence et donner voix à l'inaudible (médisances contre le roi ou l'empereur, complots de régicide, tout ce que la société n'admet pas d'entendre, comme dans la scène du jardin dans Jules César). Dans ce lieu, l'interdiction de parler laisse meurtri, tant le jardin est supposé être, dans le théâtre shakespearien, un sanctuaire où se rencontrent voix discordantes, spéculatives et tendancieuses - comme dans Richard II, où la reine, demeurée silencieuse face au jardinier bavard pendant la quasitotalité de la scène 4 de l'acte III, s'écrie, désemparée : «O, I am press'd to death through want of speaking !» (III.4.72). Lieu privilégié de la prise de parole, le jardin contribue également au déploiement de l'œuvre ; car il est cet endroit privé où se joue un drame public, voire national. Les scènes de jardins sont des épisodes intermittents qui donnent à voir les dessous de la pièce, les pensées profondes des personnages, dont la conscience se révèle en toute clarté pour donner un ton nouveau à la suite de la pièce. Il s'ensuit que certains propos peuvent sembler appropriés dans des jardins, qui sont dénoncés comme transgresseurs et traîtres dans la cité. Ainsi pourrait s'expliquer l'énervement de Plantagenet, car dans le jardin du Templier, la voix de la conscience, si loquace en général chez Shakespeare, semble en panne, se taisant là où elle devrait précisément se faire entendre.

Donner voix à ses pensées est une affaire aussi fatale aux yeux des nobles qu'elle semble vitale pour Plantagenet. Il les presse de se prononcer sur-le-champ comme si tout son être en dépendait. Le jeu de voix du personnage devient alors très complexe: dans une tension extrême et passionnelle, on l'entend aussi bien qui implore, exhorte, sollicite, ordonne, défend, dispute, accuse, dénonce, accule au choix univoque; voici un martyr qui agresse, et oppose son droit à leur devoir. Son excès de zèle, son autorité évidente, la verve exercée contre ses interlocuteurs frappés de mutisme, servent à mettre mal à l'aise autant les personnages que le spectateur :

Plan. Then say at once if I maintain'd the truth ;

Or else was wrangling Somerset in th' error?

(II.4.5-6)

Il lui faut s'en remettre aux nobles, afin qu'ils arbitrent entre sa vérité et le tort de Somerset. Sa bonne conscience si assurée réclame pourtant l'austère vérité de manière ambivalente et avec une trépidation troublante qui révèle autre chose qu'une banale insistance. Dans sa vocifération on décèle le profond tremblement 
de voix de celui que le silence abandonne devant sa propre dépossession. Somerset reformule la question de manière plus simple et plus directe, laissant le choix aux autres :

Som. Judge you, my Lord of Warwick, then, between us.

La voix a valeur d'avis; son rôle à ce stade est de formuler un jugement, d'être l'expression d'une opinion; elle sert à trancher entre celui qui détiendrait la vérité et celui qui serait en faute, entre le défendable et l'indéfendable. Exiger une voix, c'est exiger que la vérité ne demeure pas interdite mais soit véritablement dite, sous forme d'un verdict de culpabilité, lourd de responsabilités et de conséquences (voir la réponse de Vernon, vers 46-8). Or, Suffolk comme Warwick estiment cette charge trop pesante et tous deux esquivent la question d'une voix sourde et voilée, en jouant la carte de l'incompétence ou de l'équivoque :

War. Between two hawks, which flies the higher pitch ;

Between two dogs, which hath the deeper mouth ;

Between two blades, which bears the better temper;

Between two horses, which doth bear him best ;

Between two girls, which hath the merriest eye-

I have perhaps some shallow spirit of jugement;

But in these nice sharp quillets of the law,

Good faith, I am no wiser than a daw.

«We dissemble [...] vnder couert and dark speaches, when / we speake by way of riddle (Enigma)» explique George Puttenham dans The Arte of Englishe Poesie ${ }^{5}$. Si cette remarque est imputable à Talbot à la fin de la scène précédente (face à son discours énigmatique, la Comtesse remarque avoir déjà eu affaire à «a riddling merchant for the nonce» (II.3.57)), il incombe à présent à Warwick de répondre par une énigme. Sa réponse emphatique à la question de Somerset prend la forme d'une énigme qui, loin de résoudre le dilemme, le relance, le décline en de multiples métaphores chacune renvoyant comme dans un jeu de miroirs, la question de l'arbitrage dans le camp des querelleurs. Dans un mouvement de va-et-vient, cette surenchère d'images produit un ballottage sans issue. Le seul rythme alterné des phrases a quelque chose de suspect: le cumul des analogies ne représente pas une 
prise de position mais au contraire le choix d'une position stationnaire qui ressemble plutôt à une dérobade par inertie. Warwick fuit sur place. Cet étrange effet de vertige aboutit à un tour de passe-passe. C'est comme s'il cherchait à étourdir les deux rivaux par un bras de fer métaphorique.

Plus encore, il oscille dans ses métaphores entre la séduction et le danger, brassant le bien et le mal, combinant l'animalité et l'épée. (Plus loin, Somerset comparera son argument au fourreau d'une épée) ${ }^{6}$. Mais ni la hauteur du vol, ni le tranchant d'une lame, ni la puissance du galop, ni même le regard charmeur d'une femme ne suffisent pour que Warwick veuille se commettre. La voix élective demeure entre chien et loup. En effet, la seule métaphore sans ambiguïté est celle du cri du chien. Il est vrai que la voix muselée de Plantagenet, criarde, deviendra une voix assassine à travers son fils Richard. La colère gronde en lui et déjà dans ses revendications, Richard, fort de son droit, parle très haut et très fort. La voix canine des deux rivaux s'apparente à la gueule du chien qui aboie tant ils réclament leur dû en donnant de la voix. Mais par entêtement, par vertu ou par peur, Warwick est décidé à ne donner la sienne ni à l'un ni à l'autre. Si sa rhétorique est colorée et sonore, sa parole, tout compte fait, revient à une abstention de voix, procédure qui n'impressionne guère Plantagenet.

Plan. Tut, tut, here is a mannerly forbearance

Il est clair que la prise de position que recherche Richard ne tolère aucune neutralité. Si sa requête essuie une fois de plus une fin de non-recevoir, Plantagenet continue obstinément à chercher une voix à la vérité qu'il défendra becs et ongles et à gagner les nobles à sa cause. Face aux langues liées et aux voix travesties, face au sophisme de l'esquive, il en vient à user, lui aussi, à sa façon, de la ruse :

Plan. Since you are tongue-tied and so loath to speak, In dumb significants proclaim your thoughts :

Let him that is a true-born gentleman And stands upon the honour of his birth, If he suppose that I have pleaded truth, From his brier pluck a white rose with me. 
L'échec de la parole et le besoin vital de reconnaissance chez Plantagenet l'entraînent à user de stratégie en donnant du mouvement à la scène et à ses protagonistes pour combattre l'hésitation des consciences. Ayant compris l'importance emblématique du geste dans la revendication judiciaire, Plantagenet délivre astucieusement la voix du silence en exigeant qu'elle soit mimée plutôt qu'articulée. Par son initiative, une nouvelle modalité d'arbitrage s'enclenche, qui rétablit la spontanéité expressive de chacun tout en engageant ses interlocuteurs à se prononcer au nom de leur allégeance et de leur honneur. La rhétorique du geste vient suppléer celle du verbe, reléguée au second plan, et supplante la voix, finalement creuse et inefficace lorsque restreinte à sa seule oralité. Le geste libère alors la voix, dégagée de la tyrannie du langage. Plus exactement, le geste délivre le sens. Les clans se désignent par le mouvement des bras et la couleur des fleurs. Leurs convictions s'affichent à la vue de tous dans une cérémonie de paroles gesticulées. L'éloquence du silence est restituée par le geste, action que la parole ne fait qu'avaliser ; c'est cette éloquence qui occupe les devants de la scène et le champ des signifiants. C'est le signe muet que Plantagenet appelle «dumb significants», le symbole silencieux mais décisif, le geste révélateur qui permet à la vérité de transparaître symboliquement: les personnages agissent «in sign whereof... in signal of...». Dans son étude de la littérature médiévale, Paul Zumthor remarque comment

Un lien fonctionnel lie en effet à la voix le geste : comme la voix, il projette le corps dans l'espace de la performance et vise à conquérir celui-ci, à le saturer de son mouvement. Le mot prononcé n'existe pas (comme le fait le mot écrit) dans un contexte purement verbal : il participe nécessairement d'un procès général, opérant sur une situation existentielle qu'il altère en quelque façon et dont la totalité engage les corps des participants. ${ }^{7}$

Plantagenet transforme la voix en nouvel instrument théâtral et politique. La voix s'exprime par l'action, et le geste fait événement parce qu'il tend à une fin définissable : chacun prend position, se déclarant, par son mouvement, pour un parti ou pour l'autre. Ainsi la voix s'élève-t-elle au rang d'un choix de vote. D'ailleurs, le mode de scrutin adopté n'est pas sans rappeler la manière dont, à l'époque 
romaine, les sénateurs dépourvus du droit de vote exprimaient leur avis en déplaçant leur pied. Ils levaient le pied au lieu d'élever la voix. On les appelait les sénateurs pédaires. Par un tour de force remarquable, Plantagenet agit de telle sorte que ramasser une rose équivaut à taper du pied pour recueillir les voix.

Cette scène du jardin rappelle aussi l'épisode du péché originel dans la Genèse. En effet, quand Plantagenet invite les nobles à cueillir une rose d'une couleur ou d'une autre, il les place devant un choix qui exclut l'innocence - l'homme est rendu responsable de ses actes -, il déclenche une stratégie d'identification, une procédure de discrimination entre le vrai et le faux comme le fit le serpent incitant Ève à prendre la pomme de l'arbre de la connaissance. La scène du jardin du Templier représente la genèse d'une loi symbolique qui va dès lors commander la suite de la pièce. Cette brouille que les nobles voulaient maintenir entre deux individus se mue en geste allégorique qui engage tous les personnages et bouleverse le cours de l'histoire médiévale.

Maître de l'énergie des gestes et de la voix, Richard Plantagenet se révèle maître du sort, car c'est à lui somme toute que revient d'orchestrer l'ensemble de la situation. Les nobles se soumettent tous à la nouvelle règle du jeu inventée de toutes pièces et dictée par ce personnage : dépossédé de ses titres et de son héritage, il réussit néanmoins à relancer l'action de la pièce, à laquelle il donne un ton nouveau, et à gagner la voix de bien des nobles. Ironiquement, c'est au moment ou les personnages n'ont plus besoin de dire leur opinion, manifestée par un geste, qu'ils se prononcent sur la question de vérité, très clairement et cette fois sans ambages.

Suff. I pluck this red rose with young Somerset, And say withal I think he held the right.

Surtout, Shakespeare marque ici une très forte opposition entre les mots et les actes. Il suggère qu'il n'est pas possible de revenir sur l'évidence de la mise en scène du geste. Vernon formule expressément la règle fondamentale selon laquelle la voix, c'est-àdire le vote, ne se prête pas mais se donne de façon définitive, règle à laquelle Somerset et Plantagenet doivent se plier. Le vote doit être l'expression du juste et du vrai : 
Ver. Stay, lords and gentlemen, and pluck no more

Till you conclude that he upon whose side

The fewest roses from the tree are cropp'd

Shall yield the other in the right opinion.

(II.4.39-42)

C'est dans le geste d'élire qu'émerge une voix qui peut dire la vérité tant recherchée. Mais cette fois-ci la vérité appartient à la majorité des votes; non seulement ceux qui donnent leur voix doivent la respecter, mais aussi ceux qui la reçoivent comme ceux mis en échec par le vote. Son sens ne s'impose plus d'office, comme le prétendait Plantagenet. Si, avant de se prononcer, Vernon juge nécessaire de formuler la loi selon laquelle exprimer l'intention par le geste implique un engagement définitif, c'est aussi qu'il vient d'observer que la parole prononcée n'engage pas de la même manière. En effet, Warwick s'est empressé de nier la stratégie qu'il avait jusque-là décidé de suivre, exprimant son dégoût de la voix maquillée, de l'artifice et du faux-semblant, qu'il pratiquait avec tant d'habileté quelques vers plus hauts :

War. I love no colours; and without all colour

Of base insinuating flattery

I pluck this white rose with Plantagenet.

Dans celle pièce, contrairement à la suite de la tétralogie, les nobles ne reviendront plus sur leur position. Somerset et Plantagenet souscrivènt tous deux à l'exigence de Vernon, bien que de manière très différente, puisque Somerset s'engage en deux vers là ou Plantagenet se commet en deux mots :

Som Good Master Vernon, it is well objected :

If I have the fewest, I subscribe in silence.

Plan. And I.

Somerset reformule l'impératif de Vernon. Respecter le résultat des élections, c'est avant tout signer sa défaite par le silence. Il faudra apprendre à faire taire sa voix contestatrice et ne pas revenir sur la parole donnée. Le silence, comme le don de voix, devient à son tour une forme d'engagement, ce qui met Plantagenet dans une position 
embarrassante. S'il s'accorde sur ce principe, sa réponse est brève au point d'en être presque tacite et très suspecte, comme s'il se trouvait pris à son propre jeu. A ce stade Shakespeare exploite remarquablement la relation établie entre la voix et la syncope de la parole, dont le sillage tracé constitue la quintessence du théâtre. La réponse succincte de Richard est peut-être l'élément le plus révélateur de sa traîtrise future, que Somerset ne devine que trop bien, soulignant le décalage entre ce qu'expriment le corps et la voix du personnage. Il devine chez son rival colère et mauvaise foi. Somerset traversera la pièce en essayant de l'obliger à avouer ses ambitions de traître, qu'il pressent dans le discours dénonciateur et rancunier de celuici. La voix peut masquer une pensée, alors que le corps la trahit inéluctablement :

Som. No, Plantagenet,

'Tis not for fear, but anger that thy cheeks

Blush for pure shame to counterfeit our roses, And yet thy tongue will not confess thy error.

\section{$[\ldots]$}

(II.4.64-7)

Som. Your private grudge, my Lord of York, will out, Though ne'er so cunningly you smother it.

(IV.1.109-10)

Cet épisode, où est mise aux voix la vérité de Plantagenet, met également en scène la naissance du traître Richard. La trahison coule jusque dans les veines de sa gorge ; «scorn» et «malice», sentiments diaboliques que le XVIe siècle estimait sources de toute félonie, sont des termes qui rythment son discours au moment où la querelle du jardin du Temple atteint son paroxysme. Suffoquant sous la pression impitoyable d'une voix frustrée et d'une parole étouffée par le mépris et l'insulte, Richard ne peut se retenir de cracher sa peine et sa rancœur, sa bouche éclaboussante de plosives et de dentales : «How I am braved and must perforce endure it !» (II.4.115). Dans son entretien avec Mortimer mourant, il se dit «late despised Richard», et se plaint d'avoir été «ignobly us'd» (II.5.35 \& 36). Mais les plaintes se mêlent aux accusations virulentes et calomniatrices, notamment lorsqu'il rompt le silence autour de l'affaire de son père et de l'injustice perpétrée par le roi défunt. Si on ne cesse de le faire taire dans 1 Henry VI, c'est que sa voix, vibrante de la rancœur d'un déshérité, combine l'ambition à la rage. Ce sont souvent des 
personnages bienveillants qui lui conseillent de se montrer plus diplomate, prudent, patient. «Be patient, York», s'écrie Warwick à la fin de la pièce, lorsque la voix de Richard s'élève plus haut que jamais (V.4.113). Dès II.5, Mortimer, mourant, face à ce ton de voix déchaînée, l'arrête aussitôt et lui conseille la politique du silence :

Plan. But yet methinks my father's execution Was nothing less than bloody tyranny

Mor. With silence, nephew, be thou politic ;

Mortimer lui enseigne à ne pas subir le silence mais à employer la discrétion comme mesure de prudence et de survie à la cour. L'ironie dramatique veut que Plantagenet adopte, après son entrevue avec Mortimer, le silence ou, plus exactement, la politique du secret, que Suffolk prônait au début de la scène du jardin, et que Plantagenet cherchait à tout prix à briser. Réprimer les paroles et les idées de trahison ne rendent pas innocent, surtout lorsque le silence est rompu au bénéfice du public, auquel Plantagenet continuera à confier, dans ses aparte, ses intentions profondes, imaginant la mort du roi et de ses partisans. Nous voyons comment le théâtre laisse toujours un interstice où peut s'exprimer la voix de la conscience, la vérité profonde de chaque personnage.

Plan. Well, I vill lock his counsel in my breast ; And what I do imagine, let that rest.

(II.5.118-9) ${ }^{8}$

Plan. [Aside.] Plantagenet, I see, must hold his tongue, Lest it be said, 'speak, sirrah, when you should ; Must your bold verdict enter talk with lords ?' Else would I have a fling at Winchester.

(III.1.61-4)

La violence lui monte à la gorge et, dans un silence durement gardé, il crache secrètement sa colère maladive - car il est en effet question de maladie : «l'll tell thee my disease», confie-t-il à Mortimer (II.5.44) -

York. Speak, Winchester, for boiling choler chokes

The hollow passage of my poison'd voice 
By sight of these our baleful enemies.

\section{(V.4.120-2)}

Le ressentiment et la colère intestine (cette mauvaise humeur que le XVIe siècle appelle choler, humeur créée par une mauvaise digestion ${ }^{9}$, dont souffrent Hotspur ou Fluellen) ${ }^{10}$ débordent des tripes du duc d'York. De fait, la bile était souvent associée au venin ou au poison; les sécrétions brûlantes et les potions meurtrières représentaient une même forme de danger dans l'imaginaire populaire. La voix de Plantagenet regorge de bile; Richard est un personnage fielleux et même félon. Cet attribut désigne ce qu'il y a de plus trouble chez l'homme: malignité, férocité, lâcheté, ruse ${ }^{11}$. Turpis felonia dénonce avec haine et rancune un comportement motivé par la haine et la rancune. C'est Sir Edward Coke qui voyait une relation étymologique directe entre ces deux notions : «of the many conjectures proposed, the most probable is that fellone- $m$ is a derivative of the Latin fell-, fel, gall, the original sense being one full of bitterness or venom» ${ }^{12}$. Pour le poète Thomas Churchyard, il semblait évident que «sedition is a sickness and a byell» ${ }^{13}$ : 1a parole traîtresse s'engendre dans l'acrimonie et l'animosité, sentiments destructeurs. Cette explication serait d'ailleurs renforcée par l'évolution sémantique du mot en français, puisque au XVIe siècle «félonie»s'emploie pour désigner un «vomissement de bile».

Raisonne aussitôt dans ces définitions le cri d'indignation du Mayor qui, dans 1 Henry VI, s'étonne de la nature bilieuse de Winchester et de Gloucester : «Good God, these nobles should such stomachs bear !» (I.3.88). L'imaginaire shakespearien trempe souvent le traître dans le venin et l'assimile au serpent ${ }^{14}$. Dans Richard II c'est York qui assimile le premier la parole des flatteurs à des «sons vénéneux», voix que le roi «avale». C'est également Richard qui dénonce le venin des rebelles irlandais (II.1.157) ou des traîtres flatteurs qui changent de camp et rejoignent Bolingbroke. Le comte de Wiltshire, Bagot, Bushy et Green sont tous «villains, vipers, damned without redemption» (III.2.129). Avec le temps, la culpabilité du félon-traître vient se loger au tréfonds du corps, dans ses tripes, dans son cœur - surtout, dans le théâtre de Shakespeare, chez les monarques : «The king, thy sovereign, is not quite exempt / From envious malice of thy swelling heart» (1 Henry VI, III.1.25-6).

Sur les conseils de Mortimer, Richard d'York va tenter de maîtriser sa voix en intériorisant le mal qu'il trouve si dur de taire. Ce faisant, il ne deviendra pas plus prudent mais plus pernicieux, 
félon jusqu'au tréfonds de l'âme, traître inforo conscientiae. Traître par nature - par hérédité - Plantagenet trouvera dans le silence un moyen de couver ses intentions criminelles. Surtout, il ne saura plus exprimer à voix haute que ce qui le ronge intérieurement, un mal prémédité, excogitatus, intentionnel et donc criminel, de sorte que son secret sera foncièrement mal gardé. $\mathrm{Si}$, dans la scène du jardin du Templier, il fait preuve d'une certaine virtuosité de voix, il ne saura pas, comme le fera si bien son fils, doubler et multiplier les voix en lui (au point de devenir son propre commentateur), faisant que deux voix s'élèvent en une seule personne. Nous assistons toutefois dans cette pièce à la naissance de l'éloquence du fils dans le père. Dans la voix de Plantagenet/York se forge déjà la voix assassine du monstre éloquent qui lui succédera et prend forme la voix de cette offensante exception à l'humanité que sera Richard III.

Catherine LIS AK Université de Bordeaux III

\section{NOT E S}

${ }^{1}$ Nicolas Ling, Politeuphuia (1597), $\mathrm{f}^{\text {os }}$ 145-146.

21 Henry VI, II.4.1. Édition Arden (deuxième édition) établie par A.S. Cairncross, qui se fonde sur le premier folio de 1623.

${ }^{3}$ Voir Henry V, V.2.

${ }^{4}$ L'édition employée tout au long de cet article sera The Arden Shakespeare, deuxième édition.

${ }^{5}$ George Puttenham, The Arte of English Poesie, éd. G.D. Willcock \& A. Walker, Cambridge U.P., 1936, p. 188 (sig. $\mathrm{Yi}^{\mathrm{r}}$ ).

Plan. Now, Somerset, where is your argument?

Som. Here, in my scabbard, meditating that Shall dye your white rose in a bloody red. (II.4.59-61)

${ }^{7}$ Paul Zumthor, La lettre et la voix, Paris, Éditions du Seuil, 1987, p. 273.

${ }^{8}$ Voir également à ce propos la citation suivante :

War. I dare presume, sweet Prince, he thought no harm.

Plan. And if I wist he did - but let it rest. (IV.1.179-80) 
${ }^{9}$ E.M.W. Tillyard, The Elizabethan World Picture, London, 1943, p. 768.

101 Henry IV, I.3.127; Henry V, IV.7.180.

11 Voir le Oxford English Dictionary.

${ }^{12}$ Sir E. Coke, First Part of the Institutes, p. 391. Dans le Year Book 21-2 Edw. I. p. 355, un juge parle comme si dans son esprit la félonie et le venin étaient directement liés. Pour informer le pape que l'évêque d'Ely se comporte en traître, Henry III dit : «non oblitus antiquam suae mentis et fellitam malitiam» (en ne perdant pas le souvenir de l'ancienne et amère méchanceté de leur esprit). Foedera, Conventiones, Litterae etc., éd. T. Rymar, I. p. 155.

${ }^{13}$ T. Churchyard, A discourse of rebellion (1570), sig. A.iii.

14 Sur ce point, voir Richard D. Altick, «Symphonic Imagery in Richard Il», PMLA, nº2, 1967, p. 359-60 \& 365. 